\title{
Laboratoires vivants en innovation sociale et coconstruction des connaissances dans les villes : les cas du TIESS (Montréal) et de l'OBISF (Florianópolis)
}

\author{
Thiago Magalhães ${ }^{a}$, Annie Camus ${ }^{b}$, Carolina Andion, Sonia Tello-Rozas ${ }^{d}$
}

RÉSUMÉ. Le débat sur le rapport entre l'innovation sociale et les villes s'est élargi au cours des dernières décennies. Ce débat met en évidence l'intérêt suscité par les processus de coconstruction des savoirs dans les laboratoires vivants en innovation sociale (LVIS). Cet article a pour objectif de présenter une approche conceptuelle et analytique du traitement des LVIS, ainsi que de décrire et de mettre en perspective deux expériences de mise en œuvre de LVIS dans les villes : l'Observatoire de l'innovation sociale de Florianópolis (OBISF) au Brésil et Territoires innovants en économie sociale et solidaire (TIESS) à Montréal au Canada. Bien qu'ils émergent dans des réalités et des contextes différents avec des méthodologies de mise en œuvre spécifiques, qui sont présentées dans le texte, la discussion et l'analyse des deux cas apportent des pistes d'apprentissage sur les défis et les perspectives quant à la coconstruction des connaissances visant à renforcer les dynamiques d'innovation sociale à l'échelle d'une ville.

\begin{abstract}
The debate linking social innovation and cities has expanded in recent decades. This debate highlights the interest created by the processes of knowledge co-construction that take place in the Social Innovation Living L abs. This article presents a conceptual and analytical approach to SILL and also describe and put in perspective two international experiences of SILLs' implementation in cities: the Observatory of social innovation of Floriannópolis (OBISF) in Brazil and the Territoires innovants en économie sociale et solidaire (TIESS) in Montreal, Canada. Although they emerge in different realities and contexts, with specific methodologies of implementation, which are presented in the text, the discussion and analysis of the two cases provide some learning clues about the challenges and perspectives on collective learning to reinforce dynamics of social innovation in the context of the city.
\end{abstract}

La difficulté à résoudre les problèmes socioenvironnementaux complexes, l'aggravation des inégalités, les problèmes de corruption, la délégitimation du politique, le manque d'efficacité des politiques participatives et de nombreux autres phénomènes sont des symptômes de la crise actuelle des démocraties modernes, tant au nord qu'au sud (Offe, 1985; Sousa Santos, 2002; Ansell, 2011). Dans ce contexte, le débat qui met en rapport l'innovation sociale, le développement urbain et/ou territorial ainsi que la question des villes s'est élargi au cours des dernières décennies (Moulaert et Sekia, 2003;
MacCallum, Moulaert, Hillier et Haddock, 2007; McPhearson, Andersson, Elmqvist et Frantzskaki, 2015; Castelnovo, Misuraca et Salvoldelli, 2016).

Plus que jamais, il importe de mettre en place de nouveaux modèles de gouvernance, de production, de consommation et de développement, mais également de nouvelles stratégies de gestion des politiques publiques permettant de faire face aux problèmes publics complexes qui émergent avec la crise socioenvironnementale contemporaine. C'est dans cet esprit que la discussion autour des dynamiques d'innovation sociale s'intensifie, dans la mesure où

\footnotetext{
a Étudiant au doctorat en administration publique, Université d’État de Santa Catarina, Brésil et stagiaire de recherche, ESG UQAM

b Professeure, département d'organisation et ressources humaines, ESG UQAM

c Professeure, département d'administration publique, Université d'État de Santa Catarina, Brésil

d Professeure, département d'organisation et ressources humaines, ESG UQAM
} 
cette forme d'innovation est conçue comme porteuse de transformations sociales et comme vecteur de renforcement de la démocratie.

Insérés dans ces discussions, nous nous éloignons de la conception réductrice de l'innovation sociale vue comme un vecteur entrepreneurial apportant une solution ponctuelle aux problèmes sociaux pour adopter plutôt une conception de l'innovation sociale comme un processus de coconstruction et de comaitrise collective des situations problématiques. Les innovations sont ainsi liées aux dynamiques des changements sociaux et soutenues par la mobilisation et la participation de différents collectifs à la résolution de "problèmes publics » (Cefaï, 2017; Andion, Ranconi, Moraes, Gonsalves et Serafim, 2017; Andion, Alpersted et Graeff, 2020). Ces dynamiques, résultats d'actions collectives, permettent à différents groupes d'acteurs de jouer un rôle de premier plan dans la recherche de solutions, dans la création de nouvelles pratiques, procédures, répertoires d'actions, ainsi que dans la création d'une capacité critique individuelle et collective et d'une autonomie politique accrue. Dans cette optique, il devient important de se concentrer sur l'action territoriale à travers les expériences collectives et individuelles ainsi que leur influence sur l'innovation sociale, en tenant compte des conséquences sur les communautés et sur les politiques publiques.

Comme le soulignent Janin et Pecqueur (2017), les modèles de gestion et d'étude ainsi que les dispositifs utilisés pour penser les territoires et leurs dynamiques ont été créés il y a plus de 30 ans et ne répondent plus aux demandes et aux défis actuels. Axés principalement sur des modèles positivistes et sur des études quantitatives à grande échelle, ils ne permettent pas de visualiser les dynamiques et les particularités de chaque territoire. Or, les défis contemporains imposent la création de connaissances et de processus d'apprentissage afin de trouver et de résoudre les problèmes publics. Cela suppose donc des recherches systématiques pour comprendre et soutenir la création d'espaces de communication, de coopération, d'apprentissage, d'interobjectivation et de coconstruction de connaissances (Zask, 2004; Howaldt et Kopp, 2012).

C’est au début des années 2000 que se développe un intérêt grandissant pour les laboratoires vivants en innovation sociale (LVIS) en tant que lieu d'apprentissage, de cocréation et de diffusion du savoir dans les systèmes (Schuurman, 2015; Leminen et Westerlund, 2016) et, plus récemment, dans les écosystèmes d'innovation sociale (EIS) (Lévesque, 2016; Kaletka, Markmann et Pelka, 2017; Biggeri, Testi et Belluci, 2017; Alijani, Luna, Castro-Spila et Unceta, 2016). À l'origine, les laboratoires vivants étaient considérés comme une méthodologie d'innovation ouverte centrée sur l'utilisateur et le client afin de détecter, tester et valider leurs perceptions sur de nouveaux produits, en particulier ceux liés aux technologies de l'information et de la communication (Leminen et Westerlund, 2016). En 2006, un avis de soutien publié par la Commission européenne (CE) renforce cet intérêt pour les projets d'innovation basés sur la méthodologie des living labs, comme le fera aussi la mise sur pied du European Network of Living Labs (ENoLL). De cette évolution de la définition des living labs et du déploiement du réseau européen, les premières expériences centrées sur les laboratoires sociaux, citoyens et urbains (plus tard appelés laboratoires vivants en innovation sociale LVIS) ont commencé à être étudiées.

Klein, Fontan, Harrisson et Lévesque (2012) et Janin et Pecqueur (2017), en analysant des expériences de LVIS dans les contextes canadien et français, montrent que ces laboratoires peuvent constituer un outil important de l'action collective pour repenser les pratiques et les territoires en matière de politiques publiques. Selon Roux et Marron (2017), les LVIS répondent à un besoin de démocratisation de l'information et du savoir, de façon à permettre une meilleure compréhension de la réalité face aux défis et aux problèmes que posent les démocraties modernes. Plus que des concepteurs de solutions, les LVIS peuvent également être considérés comme un espace de coconstruction des problèmes et d'actions collectives sur le plan territorial, ce qui favorise la réflexion au sein des réseaux d'acteurs, met en pratique des processus cognitifs plus complexes et tient compte des différents savoirs et connaissances.

Dans cette perspective, cet article cherche à faire avancer la discussion sur les LVIS et sur leur capacité à repenser l'action collective territoriale et les dynamiques d'innovation sociale. À cette fin, nous proposons de faire converger le concept de LVIS et celui «d'enquête publique » développé par Dewey (1927) sur la base d'une analyse pragmatique du phénomène. Afin d'illustrer cette approche, deux expériences de LVIS sont présentées: le cas de Territoires innovants en économie sociale et 
solidaire (TIESS), un organisme de liaison et de transfert à Montréal, au Canada, et le cas de l'Observatoire de l'innovation sociale de Florianópolis (OBISF), au Brésil. Ces expériences démontrent les particularités des LVIS et nous permettent de réfléchir aux possibilités de favoriser les dynamiques de l'innovation sociale dans les territoires.

\section{Les laboratoires vivant en innovation sociale et territoriale : une approche pragmatique}

La littérature sur les LVIS est principalement basée sur une conception de l'innovation sociale en tant qu'entrepreneuriat social-technologique. Avec cette approche, l'accent est mis sur la dimension technologique, technique et/ou structurelle de l'innovation sociale, héritage issu du concept traditionnel d'innovation (Leminen et Westerlund, 2016). Les LVIS y sont considérés comme des organisations d'appui principalement développées par des centres de recherche, des organismes publics et des universités. Le rôle des universités et des centres de formation, ainsi que des spécialistes, est placé au cœur du dispositif. Comme le soulignent Leminen et Westerlund (2016), «cette méthodologie enrichit le nouveau processus de construction de la connaissance grâce à un travail collaboratif régulé par les participants avec le guide des enseignants, des tuteurs ou des mentors utilisant l'approche de l'innovation ouverte » (p. 15, trad. libre).

Cependant, plus récemment, certaines études ont mis en évidence les limites de ces approches, affirmant que, appliqué au contexte de l'innovation sociale, le concept de living labs nécessitait une perspective plus large permettant de prendre en compte les particularités théoriques et méthodologiques des dynamiques de l'innovation sociale (Masi, 2016). Cette problématisation se pose, compte tenu des processus d'apprentissage collaboratif requis et propres aux écosystèmes d'innovation sociale, où ces living labs se développent (Serra, 2013; Gascó, 2017), ainsi que de leurs possibilités de transformation de l'action collective et des politiques publiques dans les territoires (Janin et Pecqueur, 2017; Roux et Marron, 2017). C'est dans cette perspective que nous cherchons ici à rapprocher le concept de LVIS de la notion d'« enquête publique » développée par Dewey (1927) et à mobiliser les auteurs du champ d'études de la sociologie des problèmes publics et de la démocratie avec une approche pragmatique, dont Ansell (2011), Cefaï (2017), Chateauraynaud (2011) et Frega (2016).

Dans cette perspective, l'accent est mis sur les « problèmes publics », sur l'expérimentation démocratique et sur le développement des connaissances mis en pratique par une diversité d'acteurs qui traitent des situations problématiques sur le territoire. Pour Dewey (1927), la connaissance des problèmes éprouvés se produit par et dans l'expérience, à travers un processus d'enquête sur les problèmes réalisé par plusieurs acteurs. Cefaï (2017) propose la notion d'«enquête publique» précisément pour attirer l'attention sur les processus de résolution des problèmes publics, alors que les acteurs cherchent à les comprendre, les organiser et leur trouver des solutions. Le processus d'identification des problèmes et d'enquêtes collectives autour d'une situation problématique caractérise le processus d'enquête publique. Pour Cefaï (2017), il s'agit d'un processus d'enquête sur un problème posé, dans lequel les acteurs sont motivés par une connaissance qui leur donne un pouvoir d'action: "Doing is knowing, knowing is doing» (p. 189).

De plus, la situation problématique a le potentiel créatif de modifier des processus juridiques et politiques, ainsi que de produire «des effets qui ont un impact sur l'ordre moral - sur les usages, les croyances et les coutumes » (Cefaï, 2017, p. 190, trad. libre). Bref, le processus d'enquête, de problématisation, de publicization et d'action autour des problèmes permet de gagner en crédibilité et en légitimité, d'obtenir l'appui des pouvoirs publics et du grand public, ainsi que de générer des changements dans les structures et les institutions et de transformer les dynamiques dans les arènes publiques et dans la culture politique, en permettant l'exercice démocratique.

Sur la base de ces processus, des arènes publiques sont configurées. Les arènes publiques sont structurées autour de «terrains d'indignation » et de «laboratoires d'expérimentation » qui se manifestent autour de «situations de preuve" (épreuve) (Cefaï, 2017, trad. libre). Elles sont caractérisées comme des champs d'expérience collectifs, se concentrant sur des territoires limités ou dispersés, à mesure que des collectifs se forment et s'organisent autour d'attentes et d'expériences communes. Ces espaces publics sont donc compris comme des espaces de confrontation et de coopération dans lesquels les problèmes publics sont signifiés et partagés et où divers acteurs et 
instances, outre l'appareil étatique, s'articulent autour d'eux. Cefaï (2017) ajoute que c'est à travers ces expériences que les acteurs de l'espace public peuvent établir de nouvelles habitudes collectives, créer des connaissances, établir de nouveaux répertoires de définitions et des réponses types aux problèmes, possibles vecteurs des transformations et influençant les domaines plus généraux de la politique.

La perspective sociologique des problèmes publics, basée sur le pragmatisme, brièvement présentée ici, peut être une source d'inspiration pour comprendre les LVIS dans une perspective nouvelle. Dans cette optique, les LVIS deviennent un lieu de problématisation, de publication, de débat et de coconstruction de solutions aux problèmes publics. Ainsi, ils sont considérés comme des «laboratoires vivants» de coproduction sociale au sens strict. Dans les différentes arènes publiques de la ville, les acteurs ordinaires sont en mesure de mener des enquêtes publiques et commencent ainsi à participer à la définition des « conditions de leur vie » (Zask, 2004) et à la transformation de leur réalité.

Il est important de comprendre que ces laboratoires vivants sont configurés en tant que réseaux d'interaction entre différents acteurs qui se mobilisent et promeuvent des dynamiques d'innovation sociale, autour des principaux problèmes éprouvés à l'échelle locale. Ces réseaux, dans la littérature, sont souvent conçus comme des écosystèmes d'innovation sociale (EIS) (Lévesque, 2016; Kaletka et collab., 2017; Biggeri et collab., 2017; Alijani et collab., 2016) formés par des systèmes d'offre (ou de soutien), ainsi que comme des initiatives de plusieurs types (mouvements sociaux et autres collectifs associatifs d'économie sociale et solidaire, gouvernementaux et entrepreneuriaux) qui mettent en pratique l'innovation sociale.

Plus que des concepteurs de solutions, les LVIS peuvent également être considérés comme des espaces de coconception des problèmes et des actions sur le plan territorial, ce qui favorise la réflexion au sein des réseaux d'acteurs et la collaboration. En ce sens, ils portent un processus de coconstruction des savoirs et connaissances et de l'intelligence collective basé sur la coopération et la communication, apte à générer une «capacité d'innover» parmi les différents acteurs dans les EIS et également à constituer un important dispositif d'action publique pour repenser les pratiques et les territoires en matière de politiques publiques (Janin et Pecqueur, 2017).
Afin de comprendre comment les expériences de LVIS peuvent être vues dans l'approche présentée cidessus, nous examinons deux cas qui, à notre avis, sont probants pour réfléchir les LVIS sous l'angle des dynamiques des problèmes publics et des innovations sociales territoriales dans les villes.

\section{Les cas du TIESS à Montréal (Canada) et de l'OBISF à Florianópolis (Brésil)}

Dans cette section, nous décrivons deux cas de laboratoires vivants d'innovation sociale. Les deux expériences stimulent l'interaction entre les communautés et les universités locales autour des problèmes publics de la ville, en articulant enseignement, recherche et transfert en innovation sociale. Les deux expériences prennent place dans des agglomérations avec un territoire insulaire et qui se distinguent comme villes innovantes et greniers d'innovations sociales. En ce qui concerne Florianópolis, elle a été élue première parmi les 10 villes brésiliennes au potentiel le plus innovant par le ministère des Sciences, de la Technologie et de l'Innovation du Brésil. Montréal, quant à elle, a reçu en 2006 le titre de Ville de design créatif par l'UNESCO. Elle a aujourd'hui son quartier de l'innovation et participe au Défi des villes intelligentes.

Ainsi, les expériences du TIESS et de l'OBISF nous apparaissent représentatives de la coconstruction du savoir dans des EIS urbains. Grâce à ces deux expériences pratiques et à la collaboration de chercheurs et praticiens, il est possible de produire et de diffuser de nouvelles connaissances sur l'innovation sociale, de renforcer l'expérimentation démocratique (Ansell, 2011) et de promouvoir de nouveaux styles de développement dans les villes. En raison de ces caractéristiques, ces expériences peuvent être interprétées comme des LVIS et il est donc pertinent de les étudier pour comprendre les innovations sociales et leurs conséquences sur les territoires.

\subsection{Territoires innovants en économie sociale et solidaire (TIESS)}

Territoires innovants en économie sociale et solidaire (TIESS) est un organisme de liaison et de transfert reconnu par le gouvernement du Québec qui a pour mission de "contribuer au développement territorial par le transfert de connaissances en 
outillant les organismes d'économie sociale et solidaire afin qu'ils puissent faire face aux enjeux de société de façon innovante et transformer leurs pratiques » (TIESS, 2020, s. p.). Pour y arriver, le TIESS s'est donné trois mandats : liaison, veille et transfert. Il les réalise à travers des structures de concertation et de travail régionales, thématiques et scientifiques, où les milieux de la recherche et de la pratique élaborent et mènent conjointement divers projets et activités.

Le TIESS a été fondé en 2014 à l'initiative de quatre acteurs de l'EIS du Québec, soit le Chantier de l'économie sociale (organisme sans but lucratif de représentation), deux centres de recherche universitaires (le Centre de recherche sur les innovations sociales [CRISES] et l'Institut Karl Polanyi de l'Université Concordia) et le Service aux collectivités de l'Université du Québec à Montréal.

La cocréation du TIESS s'inscrit dans une tradition de recherche partenariale bien établie au Québec, particulièrement en économie et innovation sociales. Déjà, à la fin des années 1970, la Politique des services aux collectivités et la mise en place d'un service dédié à la mise en œuvre de cette politique à l'UQAM institutionnalisent une approche de coconstruction de connaissances en permettant aux mouvements associatifs (syndicaux, des femmes, communautaires) d'accéder aux compétences et ressources universitaires dans un véritable partage des savoirs académiques et citoyens (Huot et van Schendel, 2019).

Dans les années 2000, des structures de recherche viendront à leur tour institutionnaliser le travail conjoint des chercheurs universitaires et des praticiens en économie sociale. Il s'agit de l'Alliance de recherche universités-communautés (ARUC) et du Réseau québécois de recherche partenariale en économie sociale (RQRP-ÉS). Cependant, la diffusion des résultats des recherches menées dans le cadre de ces instances reste insuffisante, surtout dans les milieux de pratique. Comment pérenniser et valoriser les connaissances coproduites dans le cadre de ces efforts de recherche et, surtout, comment s'assurer que ces nouvelles connaissances seront intégrées de façon durable dans la pratique? C'est à ces besoins que le TIESS est venu répondre.

Le TIESS n'est donc pas un centre de recherche, mais véritablement un dispositif de liaison et de transfert entre la recherche et la pratique qui vise à assurer une diffusion et une appropriation des connaissances coconstruites par celles-ci. L'approche du TIESS reconnait d'emblée que les savoirs théoriques et pratiques se complètent :

L'importance égale accordée aux connaissances pratiques et théoriques ne repose pas sur une négation de l'importance de la recherche scientifique. Elle nait du constat que la connaissance dans le domaine de l'innovation sociale (IS) nait non pas en vase clos, mais dans l'action, dans des processus d'essais et d'erreurs à partir des pratiques. (Huot et van Schendel, 2019, p. 17)

Le TIESS regroupe aujourd'hui plus de 70 institutions d'enseignement supérieur (universités et cégeps), centres de recherche et réseaux de soutien au développement de l'économie sociale et au développement des territoires. Son succès tient à trois grands facteurs présents dès sa création : son enracinement dans l'EIS, son enracinement dans le milieu universitaire et l'habitude du travail partenarial basé sur l'expérience en recherche partenariale (Huot et van Schendel, 2019). L'enracinement dans l'écosystème est particulièrement crucial, surtout en regard des nombreux acteurs et institutions qui y sont présents, dont certains depuis plus de 20 ans. La figure 1 représente cet écosystème et les principales catégories d'acteurs qui le peuplent. Elle permet de situer le TIESS dans cette population. 


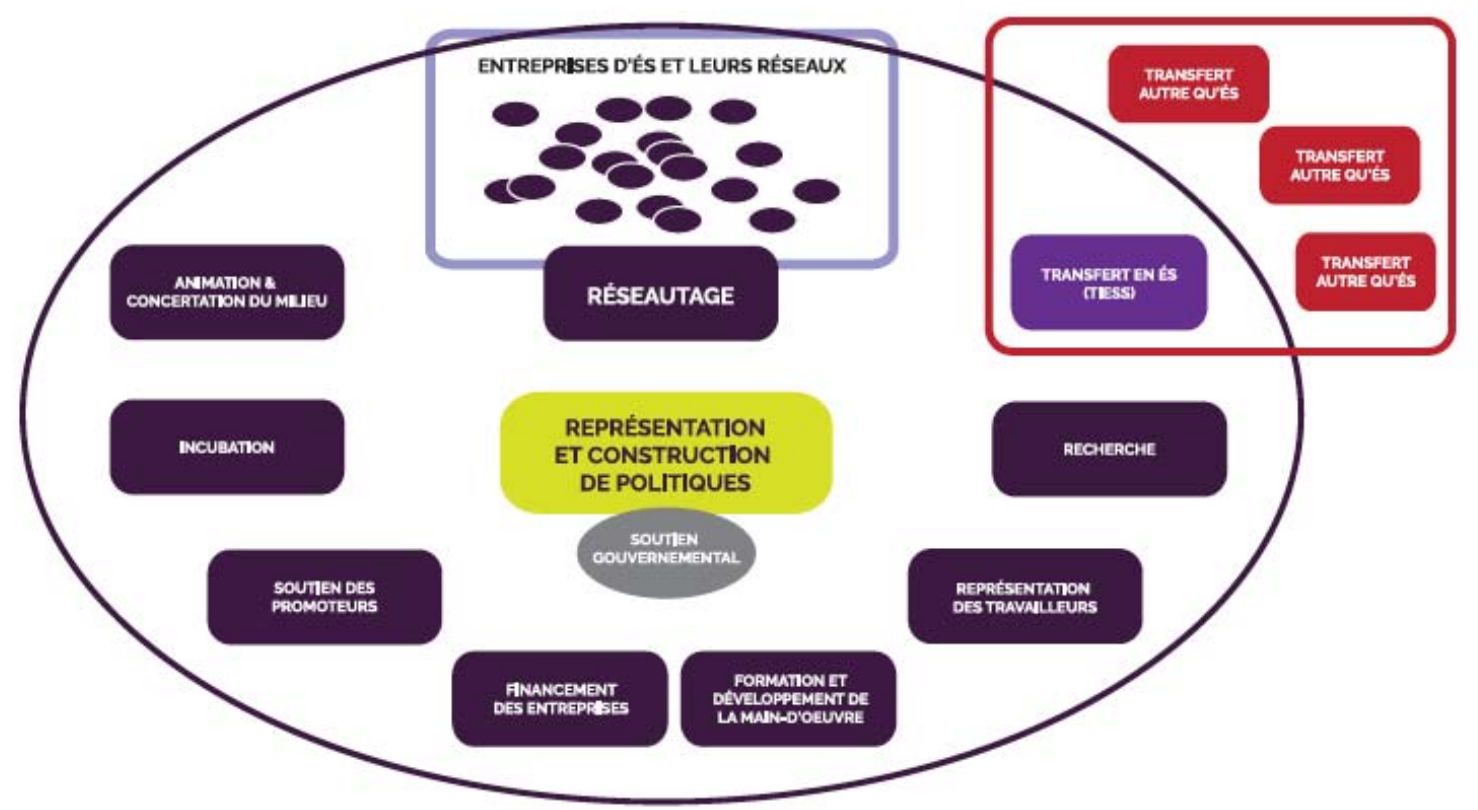

Figure 1 - Le TIESS au sein de l'EIS en éonomie sociale au Québec Source : Huot et van Schendel, 2019, p. 24

\subsubsection{La méthodologie du TIESS ${ }^{1}$}

Comme indiqué dans la section précédente, le travail du TIESS repose sur trois mandats, soit ceux de liaison, de veille et de transfert.

\section{Mandat de liaison}

À travers son mandat de liaison, le TIESS met en relation et regroupe chercheurs et praticiens autour d'un enjeu ou d'un intérêt commun, puis travaille avec eux afin de développer une compréhension commune du ou des problèmes. Ce travail passe aussi par le développement d'un langage commun et accessible à tous les membres du groupe. Le TIESS mobilise ainsi des compétences d'animation, d'écoute, d'ouverture, d'humilité, de traduction et de médiation pour soutenir le groupe dans son élaboration d'objectifs communs et de façon à maintenir un climat propice à la collaboration. La liaison suppose donc aussi de travailler à une reconnaissance égale des connaissances et expertises de chacun.

\section{Mandat de veille}

Le travail de veille cherche à déterminer quels sont ou seront les enjeux susceptibles de demander des solutions ainsi que les initiatives et expériences por- teuses et inspirantes qu'il vaudrait la peine d'explorer ou de diffuser. Pour y arriver, le TIESS compte sur son réseau d'antennes régionales et de groupes de travail ainsi que sur toutes les autres structures qui lui sont associées.

\section{Mandat de transfert}

Enfin, le travail de transfert repose au TIESS sur deux démarches intégrées :

[La] première [démarche] consiste à rendre accessibles les connaissances et les informations existantes par la production de différents outils (outils didactiques, vidéos, bulletins, articles, site web, etc.). La deuxième démarche repose sur le travail conjoint des chercheurs et des praticiens et vise l'intégration de connaissances ou de procédés nouveaux dans la pratique des individus et des organisations. (Huot et van Schendel, 2019, p. 28)

Ces trois mandats se concrétisent à travers les projets qu'accompagne ou mène le TIESS, qui fonctionne à la fois en amont et en aval de l'innovation sociale. En effet, les projets peuvent suivre deux types de parcours : 1) une coconstruction de connaissances pour mieux comprendre et systématiser des innovations sociales existantes ou 2) une 
coconstruction de connaissances qui mènera à la création d'innovations sociales. Dans les deux cas, le TIESS s'assure ensuite d'accompagner la coconstruction d'outils adaptés aux milieux et organisations ciblés, puis de faire connaitre et de transférer ces outils de façon à soutenir l'adoption la plus large possible des innovations.

Quant aux projets menés au sein du TIESS, ils se déroulent en plusieurs phases - et pas toujours d'une façon linéaire :

\section{a) Repérage}

C'est le moment qui vise à détecter les besoins. Une large partie des projets du TIESS est issue de demandes des milieux de pratique qui ont identifié un ou plusieurs besoins ou problèmes, tandis qu'une autre partie provient des chercheurs. D'autres projets proviennent du travail de veille du TIESS lui-même.

\section{b) Sélection, montage et démarrage}

Ensuite, on procède à des moments de sélection, de montage et de démarrage. Lors de ces "phases", les besoins, le ou les problèmes ciblés et les objectifs doivent être clarifiés par les acteurs. Il y a donc un travail de codesign du projet avec les différents partenaires. Ces travaux se déroulent sous plusieurs formes selon le projet : en groupe, en rencontres individuelles avec chacune des parties prenantes, etc. C'est aussi pendant ces phases que le projet est soumis à différents bailleurs de fonds pour obtenir du soutien financier.

\section{c) Innovation et expérimentation}

On parle ensuite de moments d'innovation et d'expérimentation, alors que le TIESS travaille avec les acteurs à réaliser une synthèse de connaissances autour du problème ou de l'enjeu retenu. C'est aussi à ce moment qu'il peut y avoir la tenue de projets-pilotes, à la suite desquels se déploiera le travail d'élaboration et de production d'outils visant le transfert et l'appropriation des solutions et des pistes de solution/d'action identifiées. Ces outils peuvent prendre une multitude de formes : ateliers, séminaires, groupes de codéveloppement, ou encore outils plus traditionnels (guides, cahiers, webinaires).

\section{d) Mise à l'échelle et pérennisation}

Enfin, le TIESS travaille avec les membres du projet à la diffusion des connaissances et des outils non plus auprès des participants au projet (les pionniers), mais auprès du plus grand nombre possible afin de toucher une masse critique et d'enclencher une transformation des pratiques. Cette phase implique généralement le recours à des relayeurs vers les milieux universitaires, de pratique et les pouvoirs publics. Chaque projet fait l'objet d'une phase d'évaluation.

\subsection{L'Observatoire de l'innovation sociale de Florianópolis (OBISF)}

L'OBISF est une plateforme de recherche partagée par deux groupes de recherche de l'Université d'État de Santa Catarina au Brésil : le Centre de recherche sur les innovations sociales dans la sphère publique (NISP) et le groupe Strategos. L'OBISF compte presque 20 partenaires institutionnels ${ }^{2}$ issus du gouvernement, de la société civile et du monde des affaires. Il est financé par la Fondation pour le soutien à l'innovation et à la recherche de Santa Catarina (FAPESC) et par le Conseil national pour le développement scientifique et technologique (CNPQ). Il compte sur une équipe de plus de 20 chercheurs comprenant des professeurs ainsi que des étudiants à la maitrise et au doctorat.

L'OBISF a été coconstruit, en 2017, par un projet de recherche lié à l'enseignement et au transfert. L'objectif était de mettre en place une plateforme collaborative en ligne permettant de connaître, de suivre et d'analyser l'écosystème d'innovation sociale de la ville. En d'autres termes, plus qu'une analyse structurelle de l'EIS, l'objectif était de cartographier les arènes publiques de la ville et de suivre les expériences de mobilisation, de problématisation et de construction collective de solutions à ces problèmes publics (Cefaï, 2017).

À cette fin, parallèlement à l'Observatoire, a été créé le Laboratoire d'éducation pour le développement durable et l'innovation sociale (LEDS), qui consiste en une sorte de "laboratoire vivant» destiné à accompagner et à faciliter les processus de recherche publique dans les arènes publiques retenues. À partir des expériences suivies et à la demande des acteurs, le LEDS réalise plusieurs activités (projets, événe- 
ments, ateliers, etc.) qui en font un espace de collaboration pour la coconstruction de connaissances dans l'interaction entre les connaissances produites à l'Université et celles dans l'EIS.
Compte tenu de ces aspects préliminaires, le cadre analytique et méthodologique adopté dans le projet a été structuré en quatre moments principaux, qui n'ont pas été développés de manière linéaire. Ces quatre moments sont résumés à la figure 2 , puis décrits ci-dessous.

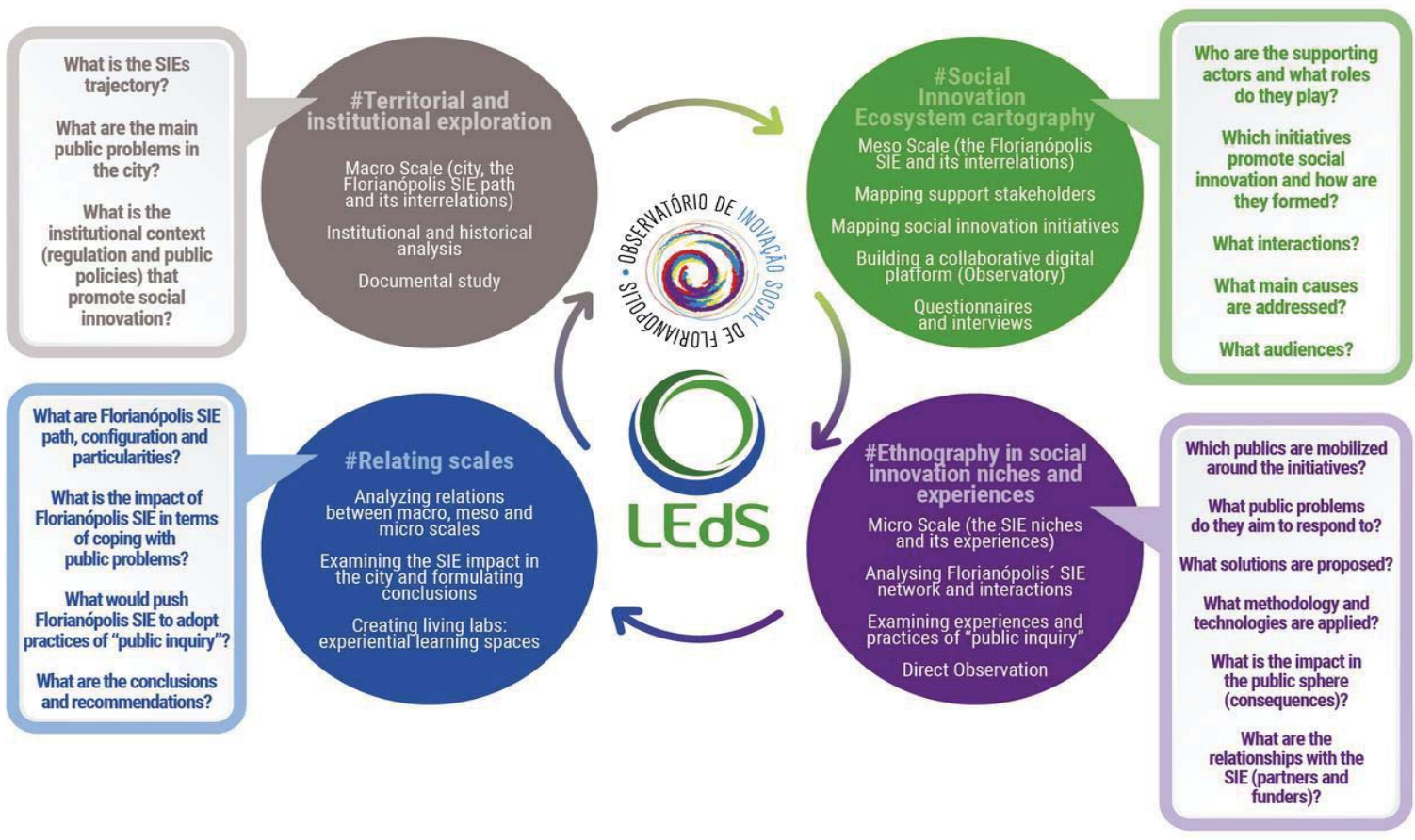

Figure 2-Cadre analytique et méthodologique Source : Observatório de Inovação Social de Florianópolis, 2020a, trad. libre

\subsubsection{Moment 1: Exploration territoriale et institutionnelle}

Ce moment commence par un examen du contexte institutionnel (lois, règlements, politiques et programmes publics) qui soutient l'innovation sociale dans la ville, par analyse documentaire et par contenu. Outre les dispositions légales, la dimension territoriale et l'histoire de l'EIS ont été prises en compte, dont son émergence et son développement, ainsi que ses dynamiques territoriales, l'accent étant mis sur l'identification des principaux problèmes publics de la ville. Afin de respecter cette étape, les principaux acteurs impliqués dans l'EIS de Florianópolis ont été interrogés et les principaux rapports traitant des défis actuels de la ville ont été analysés. Les informations préliminaires sur la métropole et ses principaux problèmes, sur le contexte institutionnel et sur la compréhension de la formation et du développement de l'EIS ont ainsi fourni un panorama à l'échelle macro, qui a été considéré ensuite pour l'analyse des échelles méso et micro.

\subsubsection{Moment 2 : Cartographie de l'écosystème d'innovation sociale}

Cette étape a débuté par des entretiens avec les principaux acteurs du système de soutien à l'innovation sociale dans la ville. À travers ces entretiens et par une méthodologie « boule de neige », l'équipe de l'OBISF a amorcé l'identification des initiatives d'innovation sociale de la métropole. Les initiatives répertoriées appartiennent à quatre secteurs principaux : public, privé, société civile et/ou universités. Les initiatives du secteur public sont généralement des programmes. Celles des universités peuvent être des programmes de recherche et de transfert. Dans le secteur privé, les initiatives sont aussi constituées de programmes de type responsabilité sociale, mais aussi de coopératives, d'entreprises sociales et d'entrepreneurs personnels. Enfin, celles de la société civile peuvent être des associations, des fondations, des collectifs informels, des programmes, des mouvements sociaux, des plateformes ou des applications. Au cours de cette étape, l'équipe de l'Observatoire a recueilli les informations 
publiques sur ces initiatives, notamment leur format juridique, leur mission, leurs publics cibles et leurs contacts pour le géoréférencement.

Une deuxième étape a débuté par l'observation des initiatives et leur mobilisation autour du problème public qu'elles tentent de résoudre, des solutions qu'elles proposent, de la manière dont elles mesurent leurs résultats, de celles qui s'engagent dans les actions, des méthodologies et des technologies qu'elles utilisent le cas échéant, de leur incidence dans la sphère publique ainsi que de leurs partenaires et bailleurs de fonds. De cette manière, toutes ces informations sur les initiatives d'innovation sociale et sur les acteurs de soutien sont devenues partie intégrante de la carte, ainsi que leurs interrelations. Elles ont constitué l'échelle d'analyse méso, qui compose la plateforme en ligne de l'Observatoire (voir Figure 3).
Après le lancement de la plateforme, les informations ont commencé à être fournies en ligne par les acteurs et par les responsables des initiatives de soutien, qui peuvent remplir eux-mêmes une fiche sur leur organisation. À tout moment, les acteurs peuvent accéder à la plateforme et actualiser leurs données. Le processus de validation reprend les données et les maintient à jour. Ainsi, l'Observatoire est devenu un outil en ligne à l'accès libre et gratuit pouvant être utilisé à la fois par les acteurs de l'EIS et par la société en général pour accéder à des informations et à des ressources relatives à l'innovation sociale dans la ville. En septembre 2019, la plateforme comptait 226 acteurs de soutien et 307 initiatives d'innovations sociales enregistrées. Parmi celles-ci, 112 ont été observées de plus près, ce qui représente 533 agents qui composent l’EIS de Florianópolis.

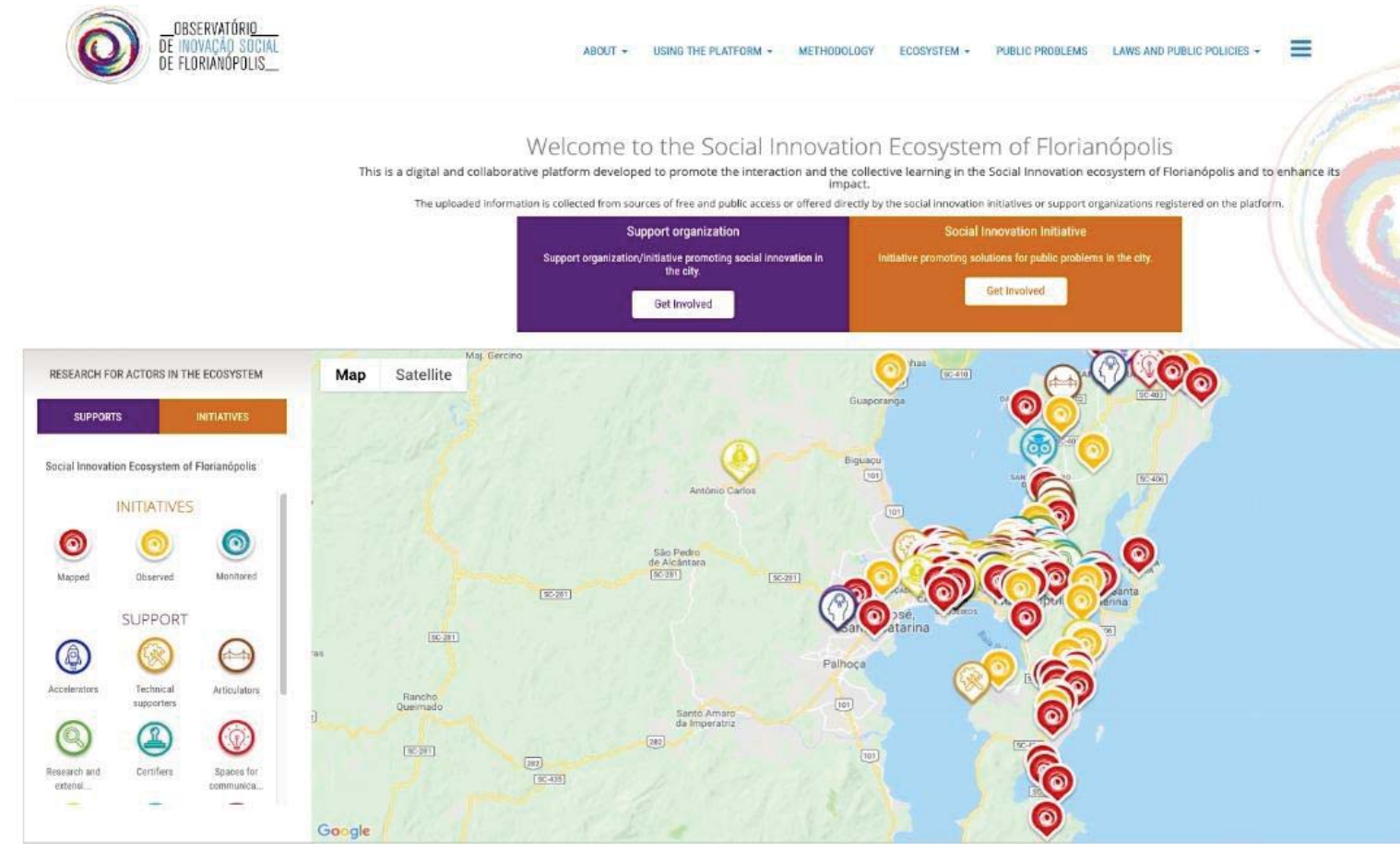

Figure 3 - Image de la plateforme en ligne Source : Observatório de Inovação Social de Florianópolis, 2020b

\subsubsection{Moment 3 : Ethnographie dans les arènes publiques}

Afin de suivre les «champs d'expérience » de l'innovation sociale à Florianópolis, des étudiants de troisième cycle mènent des études systématiques où des dynamiques d'innovation sociale sont identifiées. D'après la cartographie et les études précédemment menées par l'équipe de recherche, certaines expériences dans les arènes publiques de la ville ont été identifiées : 1) le réseau de protection des droits des enfants et des adolescents dans la municipalité; 2) le Forum municipal des politiques publiques; 3) le Réseau de traitement des déchets solides; et 4) l'articulation autour de l'agriculture urbaine. 
Le but est alors d'observer les « expérimentations » (Ansell, 2011), et non seulement des initiatives isolées. Ainsi, il est possible d'étudier plus en profondeur et à plus long terme la perception et la mobilisation des problèmes publics par les acteurs, leur mobilisation autour de ces problèmes, les solutions proposées, les méthodologies et technologies utilisées, ainsi que la portée des conséquences et les limites de ces solutions dans les arènes publiques dans lesquelles elles opèrent.

\subsubsection{Moment 4 : Rapport entre les échelles macro, méso et micro}

L'OBISF a pour objectif final de promouvoir une lecture longitudinale et multiniveau de l'EIS de Florianópolis en rapportant ses dimensions historique, territoriale et institutionnelle (échelle macro), une analyse de son réseau d'associations de formes de coopération et d'interactions (échelle méso) et les expériences et pratiques des acteurs (échelle micro). Cette méthodologie de travail permet d'observer in situ comment l'EIS se forme à l'interface des institutions déjà établies et du potentiel créatif des différents acteurs. En ce sens, l'objectif à la fin de ce travail est de consolider une sorte de laboratoire vivant en mettant l'accent sur l'apprentissage par le biais de l'expérience acquise dans les arènes publiques, puis de promouvoir les espaces de problématisation, de publication, d'exploration et d'expérimentation collective permettant de faire face aux problèmes publics de la ville.

Basé sur une analyse territoriale, multiscalaire et longitudinale, l'OBISF a permis de consolider une nouvelle approche théorique et analytique de la cartographie et de l'analyse des EIS dans les villes. Ce nouveau regard sur les EIS met en évidence la pluralité des acteurs, des rôles, des secteurs et des échelles qui composent les écosystèmes, leurs interactions et, en particulier, les processus d'« enquête publique» (Cefaï, 2017) ou des capacités générées, car la diffusion et l'impact des innovations sociales sur les EIS sont liés à des processus d'apprentissage collectif et collaboratif. Comme le soulignent Howaldt et Kopp (2012), de telles études sont pertinentes, car elles font progresser la recherche systématique pour comprendre et soutenir la création d'espaces de communication, de coopération et de coconstruction de connaissances dans les EIS.

\section{Discussion et conclusion}

\section{Quel rapport au territoire, à l'innovation territoriale et à la ville?}

Bien que les deux LVIS présentés ici se soient développés dans des contextes nettement différents, plusieurs points de convergence peuvent être soulignés. D'abord, il ne s'agit pas d'expériences isolées qui visent à donner une solution spécifique à un problème particulier. Le TIESS et l'OBISF sont inscrits dans des EIS de villes où différents acteurs de la société sont articulés et travaillent de manière conjointe dans l'identification, la problématisation, le débat et la coconstruction de solutions aux problèmes publics. En cohérence avec l'enquête sociale prônée par Zask (2004), dans ces deux espaces, des « acteurs ordinaires » sont mobilisés pour participer à des « enquêtes publiques » et pour contribuer à des processus de résolution des problèmes publics. On observe ainsi qu'il s'agit d'espaces de coconstruction dans lesquels chercheurs, praticiens et citoyens travaillent de concert et où les savoirs académiques et citoyens sont placés au même niveau.

Puis, on saisit clairement l'approche pragmatique de ces deux LVIS. Les connaissances et les solutions aux problèmes publics sont le résultat d'un processus d'essais et d'erreurs. Ainsi, les solutions sont proposées à partir de la pratique, de l'expérience et de l'expérimentation.

Enfin, les deux expériences de LVIS présentées ici font preuve d'un fort ancrage territorial local. Bien que les pratiques développées dans ces deux espaces de coconstruction puissent être transférables à d'autres contextes, l'expérimentation démocratique issue du travail d'une pluralité d'acteurs cherche des solutions et de nouvelles pistes de développement dans un territoire donné, plus étendu ou plus étroit, mais local. Par ailleurs, les deux cas font preuve d'enracinement dans des EIS.

En même temps, nous soulevons certaines différences factuelles qui distinguent les deux expériences. D'abord, la taille du territoire d'intervention; dans le cas du TIESS, l'action est étendue à une province (celle du Québec), alors que celle de l'OBISF est circonscrite à l'échelle d'une ville. Puis, quant aux liens avec le milieu universitaire, dans le cas du TIESS, ils sont développés comme une 
entité indépendante du milieu universitaire. Les bureaux du TIESS sont d'ailleurs placés physiquement hors des centres universitaires. À ce titre, et en fonction du problème public, le TIESS travaille avec plusieurs chercheurs issus de diverses universités ainsi qu'avec des collèges d'enseignement général et professionnel (cégeps). L’OBISF, pour sa part, est né à partir de l'initiative de deux centres de recherche d'une université spécifique. Il faut cependant noter que les parcours différents avec les milieux universitaires ne modifient pas le partenariat actif entre universités et milieux de la pratique dans les deux cas.

Enfin, de l'analyse exploratoire de ces expériences de LVIS développées dans des contextes nationaux et territoriaux totalement différents, nous constatons un élément crucial dans la comparaison de ces espaces de coconstruction des savoirs et des solutions collectives à des problèmes publics : il existe une pluralité des formes d'émergence, de constitution ainsi que d'approches pour la compréhension des laboratoires vivants d'innovation sociale. Dans le cas de l'OBISF, nous notons qu'il y a eu un effort préalable de coconstruction d'un cadre théorique et méthodologique ${ }^{3}$, d'inspiration pragmatique, qui a été brièvement présenté précédemment. Cette approche a été fondamentale pour orienter la cartographie et l'analyse de l'EIS de la ville, ainsi que pour mieux comprendre (dans une perspective scientifique) la portée et les limites des expérimentations en matière d'innovations sociales qui émergent dans les différentes arènes publiques de la ville.

La méthodologie du TIESS, bien qu'elle soit interprétée ici à la lumière de cette même approche pragmatique, a plutôt été inventée chemin faisant. Elle est ainsi largement inspirée de la pratique et des expériences du Service aux collectivités de l'UQAM, de l'ARUC et du RQRP-ÉS. Quand ce LVIS a été mis en marche, il n'existait pas de cadre épistémologique, analytique et méthodologique établi pour guider sa méthodologie et son organisation, bien que sa pratique (comme celles l'ayant précédée, soit le SAC, l'ARUC ou le RQRP-ÉS) s'inspire des connaissances scientifiques historiquement construites par le Centre de recherche sur les innovations sociales (CRISES), avec lequel ces expériences sont aussi liées. Ce n'est que quelque temps après sa création que le TIESS a amorcé le travail, avec ses partenaires, de formaliser et d'expliciter sa méthodologie. Il n'en reste pas moins que le rapport entre science et pratique se manifeste dans les deux cas, fût-il de formes distinctes.

Selon la définition de Lama et Origin (2006), le TIESS et l'OBISF peuvent être considérés comme des "expérimentations» de LVIS, dans la mesure où ils se fondent sur une méthodologie centrée sur les acteurs du terrain (communautés de pratique) et renforcent les "publics », si on reprend Dewey (1927) et Cefaï (2017). Les activités et la méthodologie du TIESS et de l'OBISF montrent bien un travail de valorisation des savoirs et des connaissances par les acteurs eux-mêmes. Cela implique de considérer l'interface et la rétroalimentation entre recherche et communautés; entre théorie et pratique; entre chercheurs et acteurs du terrain pendant tout le processus. Tout cela contribue à ancrer les démarches dans un contexte de vraie vie (real-life context).

Dans les deux cas, en coconstruisant une analyse territoriale de l'EIS à partir des acteurs et avec les acteurs (multiscalaire et longitudinale, concernant l'OBISF), ces recherches-actions peuvent contribuer à bâtir une nouvelle approche théorique et analytique pour la cartographie et l'analyse des dynamiques des innovations sociales dans les villes. Ce nouveau regard sur les EIS met bien en évidence la pluralité des acteurs, des rôles, des secteurs et des échelles qui composent ces écosystèmes et leurs interactions. Ces expériences doivent ainsi permettre de mieux comprendre la diffusion des innovations sociales et leur impact sur les EIS. L'étude de tels dispositifs est pertinente, tant pour faire progresser la production des connaissances que pour mieux soutenir et outiller les acteurs engagés dans ces espaces de communication, de coopération et de coconstruction que sont les EIS.

\section{NOTES}

1 Cette section s'inspire du texte Histoire et méthodologie du TIESS de Huot et van Schendel (2019).

2 Parmi eux se distinguent les représentants : 1) gouvernementaux, tels que le secrétaire de l'État de développement économique durable (SDS), la mairie de Florianópolis (PMF) et la Fundação Escola de Governo (ENA); 2) du monde des affaires, tels que l'Institut de citoyenneté entrepreneuriale (ICE); Impact Hub Floripa; Sebrae Santa Catarina, la start-up We Gov, la Fondation CERTI et l'entreprise Engie; 3) de la société civile, tels que l'Institut communautaire Grand Florianópolis (ICOM), le Forum 
municipal de politiques publiques, le Cepagro, l'Institut Vilson Groh, le Social Good Brasil et l'Institut Guga Kuerten; et 4) d'autres groupes de recherche nationaux et internationaux.

3 La conception et construction de ce cadre théorique et méthodologique est détaillé dans Andion, Alperstedt et Graeff, (2020). Il a été aussi fondé sur plusieurs études realisés, dès 2013, au sein du NISP (Andion et collab., 2017; Andion, Moraes et Gonsalves, 2017; Moraes et Andion, 2018; Gonsalves et Andion, 2019). Il a été développé en dialogue étroit avec d'autres chercheurs, projets et groupes de recherche de référence internationale dans le domaine de la recherche en innovation sociale, dont le Centre de recherche sur les innovations sociales (CRISES) au Québec ainsi que le projet SI-Drive en Allemagne (Howaldt, Kaletka, Schröder et Zirngiebl, 2018). Il faut aussi mentionner que cette approche analytique a été construite grâce au dialogue et en collaboration étroite avec des auteurs de la sociologie des problèmes publics basée sur le pragmatisme liés au Centre d'études des mouvements sociaux (CEMS) de l'EHESS à Paris (Cefaï, 2017) et aussi de la sociologie pragmatique, à travers le partenariat avec le Groupe de sociologie pragmatique et réflexive (GSPR) à l'EHESS de Paris (Chateauraynaud, 2011; Chateauraynaud et Debaz, 2017).

\section{RÉFÉRENCES}

Alijani, S., Luna, A., Castro-Spila, J. et Unceta, A. (2016). Building capabilities through social innovation: Implications for the economy and society. Finance and Economy for Society: Integrating Sustainability, 11, 293-313. https://doi.org/10.1108/S2043905920160000011016

Andion, C., Alperstedt, G. D., Graeff, J.F. (2020) Social innovation ecosystems, sustainability, and democratic experimentation: a study in Florianopolis, Brazil. Journal of Public Administration, 54(1), p. 181-200.

Andion, C., Moraes, R. et Gonsalves, A. (2017). Civil society organizations and social innovation: How and to what extent are they influencing social and political change? CIRIEC-España, Revista de Economía Pública, Social y Cooperativa, 90, 5-34. Repéré à http:/ / ciriec-revistaeconomia.es/wp-content/uploads/CIRIEC_9001_Andion_et_al.pdf

Andion, C., Ranconi, L., Moraes, L. R., Gonsalves, A. et Serafim, L. B. D. (2017). Civil society and social innovation in the public sphere: A pragmatic perspective. Revista de Administração Pública, 10(3), 40-58. http://dx.doi.org/10.1590/0034-7612143195

Ansell, C. (2011). What is democratic experiment? Contemporary Pragmatism, 9(2), 159-180. https://doi.org/10.1163/18758185-90000235

Biggeri, M., Testi, E. et Belluci, M. (2017). Enabling ecosystems for social enterprises and social innovation: A capability approach perspective. Journal of Human Development and Capabilities, 18(2), 299-306. https://doi.org/10.1080/19452829.2017.1306690

Castelnovo, W., Misuraca, G. et Savoldelli, A. (2016). Smart cities governance: The need for a holistic approach to assessing urban participatory policy making. Social Science Computer Review, 34(6), 724-739. https://doi.org/10.1177/0894439315611103

Cefaï, D. (2017). Públicos, problemas públicos, arenas públicas. Novos Estudos-CEBRAP, 36(1), 187-213. http://dx.doi.org/10.25091/s0101-3300201700010009

Chateauraynaud, F. (2011). Argumenter dans un champ de forces : essai de balistique sociologique. Paris, France : Petra.

Chateauraynaud, F. et Debaz, J. (2017). Aux bords de liviréversible : sociologie pragmatique des transformations. Paris, France : Petra.

Dewey, J. (1927). The public and its problems. Chicago, IL: Swallow Press.

Frega, R. (2016). Qu'est-ce qu'une pratique? Dans F. Chateauraynaud et Y. Cohen (dir.), Histoires pragmatiques (p. 321-347). Paris, France : Éditions de l'EHESS.

Gascó, M. (2017). Living labs: Implementing open innovation in the public sector. Government Information Quarterly, 34(1), 90-98. https://doi.org/10.1016/j.giq.2016.09.003

Gonsalves, A. et Andion, C. (2019). Ação pública e inovação social : Uma análise do sistema de garantia de direitos da criança e do adolescente de Florianópolis-SC. Revista Organizações \& Sociedade, 26(89), 221-248. http://dx.doi.org/10.1590/1984-9260892

Howaldt, J., Kaletka, C., Schröder, A. et Zirngiebl, M. (2018). Atlas of social innovation: New practices for a better future. Dortmund, Allemagne : TU Dortmund University.

Howaldt, J. et Kopp, R. (2012). Shaping social innovation by social research. Dans H. W. Franz, J. Hochgerner et J. Howaldt (dir.), Challenge social innovation: Potentials for business, social entrepreneurship, welfare and civil society (p. 43-55). New York, NY: Springer.

Huot, G. et van Schendel, V. (2019). Histoire et méthodologie des Territoires innovants en économie sociale et solidaire (TIESS). Montréal, QC : TIESS. 
Janin, C. et Pecqueur, B. (2017). Les living labs : remise en question des processus de mise en marché et de politique publique. Revue canadienne des sciences régionales, 40(1), 5-11. Repéré à www.cjrs-rcsr.org/V40/cjrstcsr40-1-1Janin-pecqueur.pdf

Kaletka, C., Markmann, M. et Pelka, B. (2017). Peeling the onion: An exploration of the layers of social innovation ecosystems Modelling a context sensitive perspective on driving and hindering factors for social innovation. European Public Social \& Social Innovation Review, 1(2), 83-93. https://doi.org/10.31637/epsir.16-2.3

Klein, J.-L., Fontan, J.-M., Harrisson, D. et Lévesque, B. (2012) The Quebec system of social innovation: A focused analysis on the local development field. Finisterra, 47(94). https://doi.org/10.18055/Finis2679

Lama, N., et Origin, A. (2006). Innovation ecosystems: Services engineering \& living labs - A dream to drive innovations. Repéré à https://atos.net/en

Leminen, S. et Westerlund, M. (2016). Living labs as open innovation networks. Technology Innovation Management Review, 2(9), 6-11. https://doi.org.10.22215/timreview/602

Lévesque, B. (2016). Économie sociale et solidaire et entrepreneur social : vers quels nouveaux écosystèmes? Revue Interventions économiques, 54. Repéré à http://journals.openedition.org/interventionseconomiques/2802

MacCallum, D., Moulaert, F., Hillier, J. et Haddock, S. V. (2007). Social innovation and territorial development. Farnham, R.-U. : Ashgate Publishing.

Masi, S. (2016). Social labs: Identifying Latin American living labs. Humanities and Social Sciences, 4(3), 76-82. https://doi.org/10.11648/j.hss.20160403.12

McPhearson, T., Andersson, E., Elmqvist, T. et Frantzskaki, N. (2015). Resilience of and through urban ecosystem services. Ecosystem Services, 12, 152-156. https://doi.org/10.1016/j.ecoser.2014.07.012

Moraes, R. et Andion, C. (2018). Civil society and social innovation in public arenas in Brazil: Trajectory and experience of the Movement Against Electoral Corruption (MCCE). Voluntas, 29(4), 801-818. https://doi.org/10.1007/s11266-017-9867-8

Moulaert, F. et Sekia, F. (2003). Territorial innovation models: A critical survey. Regional Studies, 37(3), 289-302. https://doi.org/10.1080/0034340032000065442

Observatório de Inovação Social de Florianópolis. (2020a). Metodologia. Repéré à www.observafloripa.com.br/is-page//methodology

Observatório de Inovação Social de Florianópolis. (2020b). Welcome to the Social Innovation Ecosystem of Florianópolis. Repéré à www.observafloripa.com.br/is-home?language=us

Offe, C. (1985). New social movements: Challenging the boundaries of institutional politics. Social Research, 52(4), 817-868. Repéré à http://voidnetwork.gr/wp-content/uploads/2016/09/New-Social-Movements.-Challenging-the-boundaries-ofinstitutional-politics-by-Claus-Offe.pdf

Roux, E. et Marron, Q. (2017). Les livings labs, de nouveaux dispositifs d'action publique pour penser les métropoles et les territoires. Revue canadienne des sciences régionales, 40(1), 33-41. Repéré à www.cjrs-rcsr.org/V40/cjrsrcsr40-1-4Roux-marron.pdf

Schuurman, D. (2015). Living labs: Concepts, tools and cases. Info, 17(4). http://dx.doi.org/10.2139/ssrn.2642754

Serra, A. (2013). Tres problemas sobre los laboratorios ciudadanos: Una mirada desde Europa. Revista Iberoamericana de Ciencia, Tecnologia Y Sociedad, 8(23), 283-298. Repéré à www.redalyc.org/articulo.oa?id=92427464016

Sousa Santos (de), B. (2002, 22 août). The processes of globalization. Eurozine, 68(14). Repéré à www.eurozine.com/the-processes-ofglobalisation

Territoires innovants en économie sociale et solidaire (TIESS). (2020). Le TIESS en bref. Repéré à https:/ / tiess.ca/qui-sommesnous/le-tiess-en-bref

Zask, J. (2004). L'enquête sociale comme inter-objectivation. Raisons pratiques, 15(1), 141-165. Repéré à https://scholar.google.com/scholar?hl=ptBR\&as_sdt=0\%2C5\&q=L $\% 27$ enqu $\% C 3 \% A A t e+$ sociale + comme + interobjectivation $+\% 282004 \% 29 \& b \operatorname{bnG}=$ 